\title{
Brain Tumor Segmentation from MRI Data Using Ensemble Learning and Multi-Atlas*
}

\author{
Tímea Fülöp, Ágnes Győrfi, Béla Surányi, Levente Kovács and László Szilágyi
}

\begin{abstract}
Atlases are frequently employed to assist medical image segmentation with prior information. This paper introduces a multi-atlas architecture that is trained to locally characterize the appearance (average intensity and standard deviation) of normal tissues in various observed and computed data channels of brain MRI records. The multiple atlas is then deployed to enhance the accuracy of an ensemble learning based brain tumor segmentation procedure that uses binary decision trees. The proposed method is validated using the low-grade tumor volumes of the BraTS 2016 train data set. The use of atlases improve the segmentation quality, causing a rise of up to $1.5 \%$ in average Dices scores.
\end{abstract}

Index Terms-Atlas-based image segmentation, multi-atlas, brain tumor segmentation, magnetic resonance imaging.

\section{INTRODUCTION}

Atlases and multi-atlases employed in medical image segmentation problems attempt to enhance the quality of the outcome using prior information regarding the object (organ) being segmented. Without atlases and shape models, segmentation methods can only use global and local properties of pixels (or voxels), like intensity distributions and textures. The use of atlases enable us to add further information to the segmentation process, for example, what is usually present in the same place in other similar image records, or what intensities are usually present in the same place in other normal records.

Atlases have recently been involved in several medical imaging problems, including the segmentation of brain tissues and lesions [1], [2], [3], prostate [4], lung [5], cardiac structures (e.g. myocardium) [6], [7], pancreas [8], [9], bones [10], cartilage [11], and multiple abdominal organ [12]. Atlases are used in segmentation problems based on

*This project was supported in part by the Sapientia Institute for Research Programs. This project has received funding from the European Research Council (ERC) under the European Unions Horizon 2020 research and innovation programme (grant agreement No 679681). The work of T. Fülöp was supported by the Collegium Talentum 2019 Programme of Hungary. The work of L. Szilágyi was supported by the Hungarian Academy of Sciences through the János Bolyai Fellowship program.

T. Fülöp and B. Surányi are with Dept. of Mathematics-Informatics, Sapientia University, Calea Sighişoarei 1/C, 540485 Tîrgu Mureş, Romania (phone: +40-265-206-210; fax: +40-265-206-211; e-mail \{fuloptimea1427, bela.suranyi\} at gmail.com).

Á. Győrfi, L. Kovács and L. Szilágyi are with University Research, Innovation, and Service Center (EKIK), Óbuda University, Bécsi út 96/b, H-1034 Budapest, Hungary (phone/fax: +36-1-6665585; e-mail: gyorfi.agnes at phd.uni-obuda.hu, \{kovacs.levente, szilagyi.laszlo\} at

nik. uni-obuda.hu).

Á. Győrfi and L. Szilágyi are also with Dept. of Electrical Engineering, Sapientia University, Calea Sighişoarei 1/C, 540485 Tîrgu Mureş, Romania (phone: +40-265-206-210; fax: +40-265-206-211; e-mail: \{gyorfiagnes, lalo\} at ms.sapientia.ro). image data originating from virtually all imaging modalities, including magnetic resonance images (MRI) [1], [2], [4], computed tomography (CT) [5], [9], [10], CT angiography [6], positron emission tomography (PET) [7], X-ray [13] and mammography [14]. A systematic review of earlier image segmentation solutions based on atlases and multi-atlases is given by Cabezas et al. [15]. Several earlier atlas-based solutions are summarized in the review paper of Gordillo et al. [16]. A more recent summary of such methods can be found in the work of Sun et al. [17].

This paper proposes an atlas-based extension for a brain tumor segmentation procedure that uses ensemble learning with binary decision trees. Multiple atlases are built, one for each feature, using only the normal voxels of the train data. The atlases will be able to say, what is the normal average intensity and the standard deviation of intensities at any position within the volume. These atlas values are used to update all feature values before proceeding to the training and testing of the decision tree ensemble. Numerical experiments will be performed to show the benefits brought by the multi-atlas approach, and to establish the best parameters of the atlas.

The rest of this paper is structured as follows: Section II presents the proposed segmentation method with full details on how the atlases are built and applied. Section III describes the experimental validation of the proposed methods. Section IV concludes the study.

\section{MATERIALS AND METHODS}

We start from an existing brain tumor segmentation procedure [18], [19], which is based on ensemble learning using binary decision trees (BDT) [20]. The BDTs are trained to separate voxels belonging to focal lesions from those that represent normal brain tissues. The separation relies on four observed and 100 computed features. The accuracy of the segmentation is evaluated statistically, the main accuracy indicator being the Dice score, which penalised the misclassification of both positive and negative voxels. The main goal of this study is to prove that adding prior information to the segmentation procedure using atlases can improve the accuracy of segmentation.

\section{A. Data}

The MICCAI Brats 2016 train data set contains 220 highgrade (HG) and 54 low-grade (LG) tumor volumes [21]. This study uses the whole set of LG tumor data. All volumes have the same size, consisting of 155 slices, each of which contain $240 \times 240$ voxels. Each record contains four data 
channels (T1, T2, T1C, FLAIR) that represent the observed features for all voxels. Each voxel has a label established by human experts, which is used as ground truth in our study. All data channels were registered to the T1 data using a standard automatic method and all other non-brain tissues were removed from all volumes [21]. LG tumor volumes were randomly divided in two equal groups. These groups took turns in serving as train and test data.

\section{B. The segmentation procedure without atlas}

The tumor segmentation procedure we start from was previously described in details in our previous paper [18]. MRI volumes were processed in several steps as listed below:

1) The first step is the so-called preprocessing, which is composed of the following tasks: (1) compensation of intensity inhomogeneity [22], [23], [24] using an enhanced N3 method [25]; (2) histogram normalization to provide comparable intensities on all data channels within the interval $\left[\alpha_{0}, \beta_{0}\right]$, accomplished by a context sensitive linear transform whose details are given in our previous work [26].

2) The expected correlation between neighbor voxels, and the imperfection of the automatic registration processes applied to align the four observed data channels motivate the feature generation that follows the preprocessing. Feature generation equally applies to all four observed data channels. Generated features include: (1) average, minimum and maximum extracted from spatial $3 \times 3 \times 3$-sized neighborhood; (2) average and median values extracted from planar neighborhoods with sizes ranging from $3 \times 3$ to $11 \times 11$; (3) gradient values extracted from $7 \times 7$-sized neighborhood in four different directions; (4) Gabor wavelet values extracted from $11 \times 11$-sized neighborhood in eight different directions. Together with the four observed ones, the total number of features becomes $N_{\varphi}=104$.

3) An ensemble learning approach is employed to accomplish the classification of voxels, built from binary decision trees. A number of $n_{T}=125$ decision trees are trained to separate normal and tumor voxels of the train data set. Each tree is trained using $N$ randomly selected voxels of the train data, out of which $p_{N}=93 \%$ are negatives. The procedure was tested with values of parameter $N$ ranging from 10 thousand (10k) to one million (1000k). Each non-leaf node of the decisions trees learns to make an optimal decision comparing a certain feature with a threshold, which were established by entropy based criterion. During the ensemble testing, each voxel of the test dataset receives a vote from each decision tree of the ensemble. The majority of the votes define the intermediary labeling of the test voxels.

4) Post-processing reevaluates the intermediary label of each test voxel using a morphological criterion: it compares the rate of positive intermediary voxels within a $11 \times 11 \times 11$ neighborhood of the current voxel with a previously defined threshold. Voxels with frequent positive neighbors are declared positive, regardless to the own intermediary labeling. Post-processing leads to regularized tumor shapes and improved accuracy indicator values.

\section{The multi-atlas approach}

Let us denote the whole set of MRI volumes involved in this study by $\Gamma$, the set of train volumes by $\Gamma^{(1)}$, and the set of test volumes by $\Gamma^{(2)}$. Obviously, $\Gamma=\Gamma^{(1)} \cup \Gamma^{(2)}$, and $\Gamma^{(1)} \cap \Gamma^{(2)}=\Phi$. Further on, $\Omega_{i}^{(1)}$ will stand for the $i$ th train volume, $i=1 \ldots\left|\Gamma^{(1)}\right|$, which contains $\left|\Omega_{i}^{(1)}\right|$ voxels with intensities $\omega_{i k}^{(1)} \in\left[\alpha_{0}, \beta_{0}\right], k=1 \ldots\left|\Omega_{i}^{(1)}\right|$. Analogously, $\Omega_{j}^{(2)}$ will stand for the $j$ th test volume, $j=1 \ldots\left|\Gamma^{(2)}\right|$, which contains $\left|\Omega_{j}^{(2)}\right|$ voxels with intensities $\omega_{j k}^{(2)} \in\left[\alpha_{0}, \beta_{0}\right]$, $k=1 \ldots\left|\Omega_{j}^{(2)}\right|$. Let us further denote by $\mathcal{N}$ the set of all voxels that are negative by grand truth.

In this study we propose and evaluate a multi-atlas approach that provides additional local information to each of the observed and computed features. In this order, for each feature we build an atlas based on the intensity values of normal voxels of the train data set. An important parameter that controls the size of the atlases is denoted by $S$. The proposed method was evaluated for various values of this parameter, ranging from 60 to 120 . In fact, the atlas built for any of the 104 features represents a 3-dimensional array of size $(2 S+1) \times(2 S+1) \times(2 S+1)$. Let us denote the set of possible atlas coordinates in any of the three main directions by $\mathcal{S}$, so $\mathcal{S}=\{-S,-S+1, \ldots 0 \ldots, S-1, S\}$. The atlas for feature number $\varphi\left(\varphi=1 \ldots N_{\varphi}\right)$ will be a function $A_{\varphi}: \mathcal{S}^{3} \rightarrow \mathbb{R}^{3}$, which for any point $\hat{P} \in \mathcal{S}^{3}$ situated at coordinates $(\hat{x}, \hat{y}, \hat{z})$ gives the triple $\left(\hat{\mu}_{\hat{P}}, \hat{\sigma}_{\hat{P}}, \hat{\nu}_{\hat{P}}\right)$ which represent an average intensity, a standard deviation of intensities, and the number of values used to compute the previous two, respectively.

One more definition is needed before explaining the atlas building process. For any atlas point $\hat{P}$ of coordinates $(\hat{x}, \hat{y}, \hat{z})$ we define its cubic neighborhood

$$
\begin{gathered}
\mathcal{C}_{\delta}(\hat{P})=\left\{(\hat{\alpha}, \hat{\beta}, \hat{\gamma}) \in \mathcal{S}^{3},|\hat{\alpha}-\hat{x}| \leq \delta \quad \wedge\right. \\
\wedge|\hat{\beta}-\hat{y}| \leq \delta \quad \wedge \quad|\hat{\gamma}-\hat{z}| \leq \delta\}
\end{gathered},
$$

where $\delta$ is a small positive integer, typically one ore two.

We need to define a function for each volume that maps it onto the atlas. For the $i$ th train volume $\Omega_{i}^{(1)}$, this function is defined as $f_{i}: V \rightarrow \mathcal{S}^{3}$, where $V$ describes the definition domain of the original volumes, $V=[0 \ldots 239] \times$ $[0 \ldots 239] \times[0 \ldots 154]$. We will use these mapping functions to find the corresponding atlas position for all brain pixels. To build these mapping functions we need to compute the following:

$$
\left(\begin{array}{c}
\mu_{x}^{(i)} \\
\mu_{y}^{(i)} \\
\mu_{z}^{(i)}
\end{array}\right)=\frac{1}{\left|\Omega_{i}^{(1)}\right|} \sum_{(x, y, z) \in \Omega_{i}^{(1)}}\left(\begin{array}{c}
x \\
y \\
z
\end{array}\right),
$$




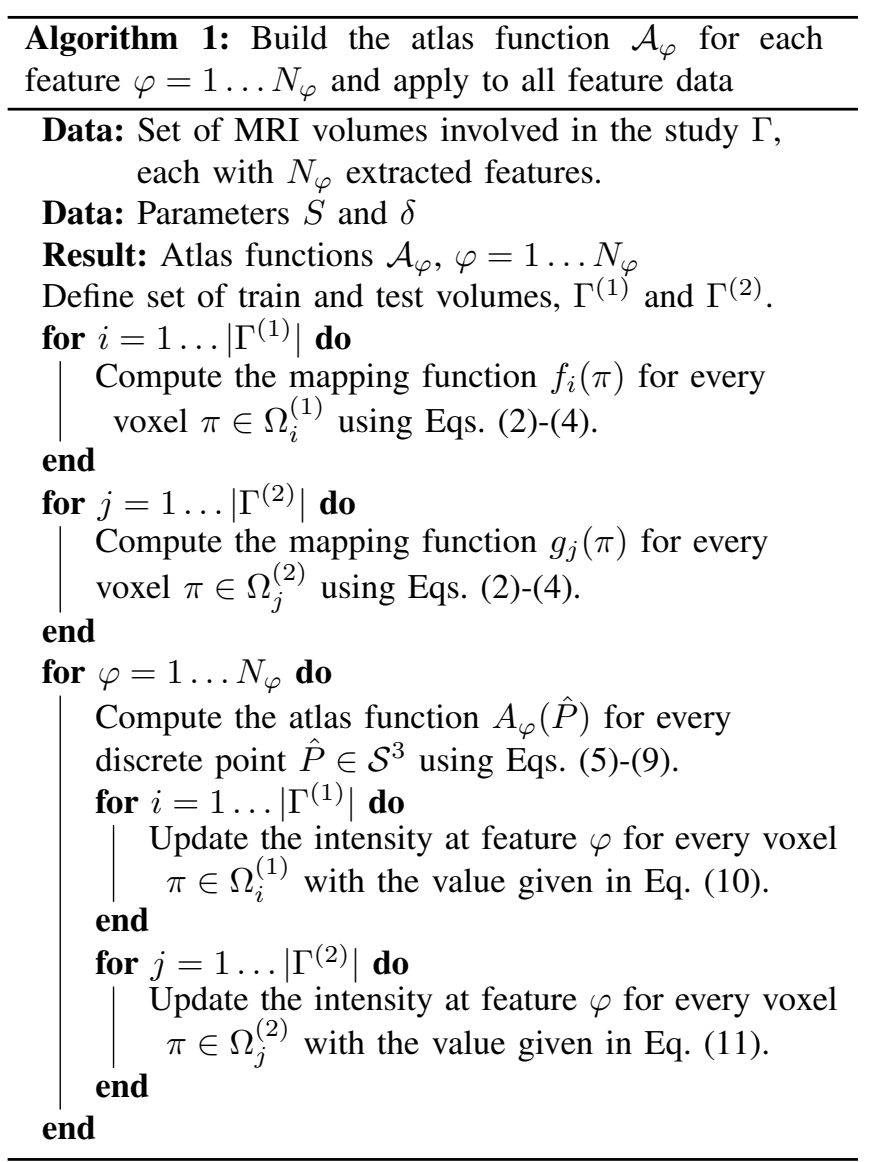

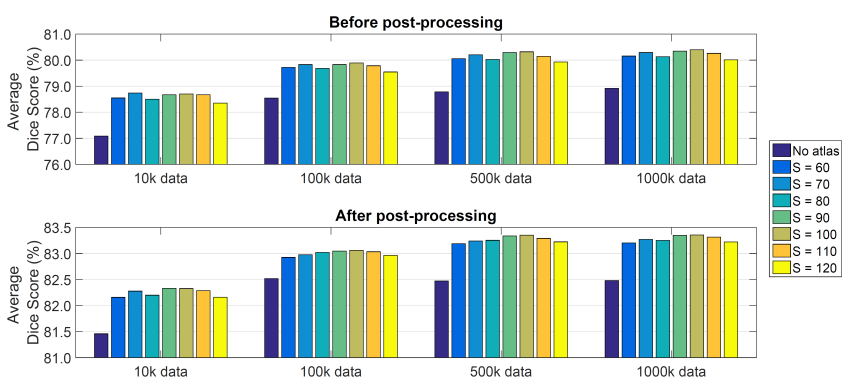

Fig. 1. Average Dice scores obtained for the 54 LG tumor volumes, before and after post-processing, without atlas and with atlas of size ranging in the set $S \in\{60,70, \ldots, 120\}$. The introduction of the atlas improves all $\overline{D S}$ values by approximately $1.5 \%$. The highest average Dice scores are achieved using the atlas of size $S=100$.

which for any $\hat{P} \in \mathcal{S}^{3}$ is established with the following formulas:

$$
\begin{gathered}
\hat{\nu}_{\hat{P}}=\sum_{i=1}^{\left|\Gamma^{(1)}\right|} \sum_{\pi \in \Psi_{i, \hat{P}}^{(1)}} 1, \\
\hat{\mu}_{\hat{P}}=\frac{1}{\hat{\nu}(\hat{P})} \sum_{i=1}^{\left|\Gamma^{(1)}\right|} \sum_{\pi \in \Psi_{i, \hat{P}}^{(1)}} \omega_{i \pi}^{(1)}, \\
\hat{\sigma}_{\hat{P}}=\sqrt{\frac{\sum_{i=1}^{\left|\Gamma^{(1)}\right|} \sum_{\pi \in \Psi_{i, \hat{P}}^{(1)}}\left(\omega_{i \pi}^{(1)}-\hat{\mu}_{\hat{P}}\right)^{2}}{\hat{\nu}_{\hat{P}}-1}},
\end{gathered}
$$

where

$$
\Psi_{i, \hat{P}}^{(1)}=\left\{p \in \Omega_{i}^{(1)}, f_{i}(p) \in \mathcal{C}_{\delta}(\hat{P}) \wedge \Omega_{i}^{(1)}(\pi) \in \mathcal{N}\right\}
$$

The intensity values of each voxel $\pi \in \Omega_{i}^{(1)}(i=$ $\left.1 \ldots\left|\Gamma^{(1)}\right|\right)$ in the train data set, denoted by $\omega_{i \pi}^{(1)}$, is updated with the value given by the formula:

$$
\min \left\{\max \left\{\alpha_{0},\left\langle\bar{\mu}+\bar{\sigma} \frac{\omega_{i \pi}^{(1)}-\hat{\mu}\left(f_{i}(\pi)\right)}{\hat{\sigma}\left(f_{i}(\pi)\right)}\right\rangle\right\}, \beta_{0}\right\} \text {. }
$$

Recommended parameter values are $\bar{\mu}=\left(\alpha_{0}+\beta_{0}\right) / 2$ and $\bar{\sigma}=\left(\beta_{0}-\alpha_{0}\right) / 10$.

The intensity values of each voxel $\pi \in \Omega_{j}^{(2)}(j=$ $\left.1 \ldots\left|\Gamma^{(2)}\right|\right)$ in the test data set, denoted by $\omega_{j \pi}^{(2)}$, is updated according to the atlas functions created for the train data set, with value given by the formula:

$$
\min \left\{\max \left\{\alpha_{0},\left\langle\bar{\mu}+\bar{\sigma} \frac{\omega_{j \pi}^{(2)}-\hat{\mu}\left(g_{j}(\pi)\right)}{\hat{\sigma}\left(g_{j}(\pi)\right)}\right\rangle\right\}, \beta_{0}\right\} \text {. }
$$

Updated intensity values for all voxels and all features are fed to the very same training and testing process presented in Section II-B.

The whole process of construction and usage of the multiatlas is summarized in Algorithm 1. 
TABLE I

AVERAGE VALUES OF ACCURACY INDICATORS IN CASE OF VARIOUS SIZES OF THE TRAIN DATA SET, OBTAINED WITH NO ATLAS OR WITH ATLAS OF DIFFERENT SIZES.

\begin{tabular}{l|c|cccccccc}
\hline Accuracy & Data & \multicolumn{7}{|c}{ Atlas size } \\
indicator & size & no atlas & $S=60$ & $S=70$ & $S=80$ & $S=90$ & $S=100$ & $S=110$ & $S=120$ \\
\hline & $10 \mathrm{k}$ & 81.458 & 82.159 & 82.278 & 82.200 & $\mathbf{8 2 . 3 3 3}$ & 82.326 & 82.288 & 82.160 \\
Average & $100 \mathrm{k}$ & 82.519 & 82.926 & 82.975 & 83.020 & 83.048 & $\mathbf{8 3 . 0 6 2}$ & 83.033 & 82.964 \\
Dice score & $500 \mathrm{k}$ & 82.473 & 83.187 & 83.241 & 83.253 & 83.336 & $\mathbf{8 3 . 3 5 2}$ & 83.290 & 83.223 \\
& $1000 \mathrm{k}$ & 82.480 & 83.203 & 83.270 & 83.250 & 83.348 & $\mathbf{8 3 . 3 5 4}$ & 83.313 & 83.221 \\
\hline & $10 \mathrm{k}$ & 83.526 & 83.993 & 84.101 & 84.045 & 84.169 & $\mathbf{8 4 . 1 7 3}$ & 84.115 & 84.004 \\
Overall & $100 \mathrm{k}$ & 84.320 & 84.584 & 84.605 & 84.642 & 84.673 & $\mathbf{8 4 . 6 8 8}$ & 84.649 & 84.563 \\
Dice score & $500 \mathrm{k}$ & 84.322 & 84.721 & 84.774 & 84.764 & 84.857 & $\mathbf{8 4 . 8 7 3}$ & 84.818 & 84.731 \\
& $1000 \mathrm{k}$ & 84.346 & 84.784 & 84.808 & 84.774 & 84.864 & $\mathbf{8 4 . 8 8 1}$ & 84.830 & 84.740 \\
\hline & $10 \mathrm{k}$ & 83.480 & 83.432 & $\mathbf{8 3 . 5 9 8}$ & 83.365 & 83.431 & 83.466 & 83.507 & 83.263 \\
Average & $100 \mathrm{k}$ & $\mathbf{8 5 . 3 3 2}$ & 84.967 & 85.005 & 84.884 & 84.987 & 85.034 & 84.978 & 84.813 \\
Sensitivity & $500 \mathrm{k}$ & $\mathbf{8 6 . 2 6 6}$ & 85.760 & 85.846 & 85.695 & 85.907 & 85.957 & 85.831 & 85.688 \\
& $1000 \mathrm{k}$ & $\mathbf{8 6 . 8 6 0}$ & 86.265 & 86.351 & 86.224 & 86.400 & 86.454 & 86.380 & 86.179 \\
\hline & $10 \mathrm{k}$ & 98.775 & 98.832 & 98.832 & 98.844 & $\mathbf{9 8 . 8 5 7}$ & 98.854 & 98.840 & 98.848 \\
Average & $100 \mathrm{k}$ & 98.733 & 98.774 & 98.773 & $\mathbf{9 8 . 7 9 2}$ & 98.786 & 98.782 & 98.782 & 98.787 \\
Specificity & $500 \mathrm{k}$ & 98.638 & 98.715 & 98.715 & $\mathbf{9 8 . 7 3 1}$ & 98.723 & 98.719 & 98.724 & 98.725 \\
& $1000 \mathrm{k}$ & 98.575 & 98.669 & 98.670 & $\mathbf{9 8 . 6 7 7}$ & 98.675 & 98.669 & 98.670 & $\mathbf{9 8 . 6 7 7}$ \\
\hline
\end{tabular}

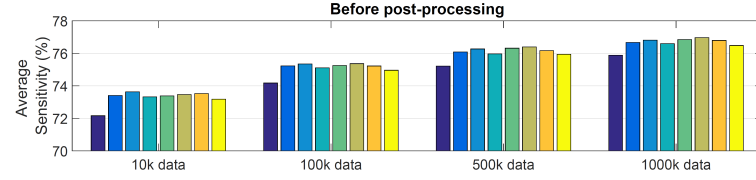

After post-processing

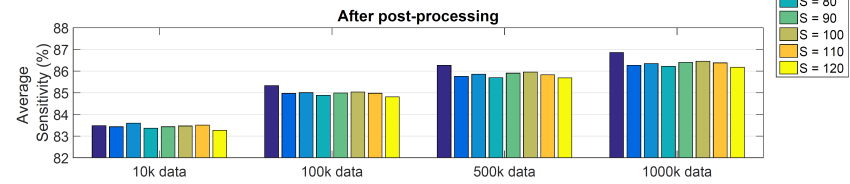

Fig. 2. Average Sensitivity values obtained for the 54 LG tumor volumes, before and after post-processing, without atlas and with atlas of size ranging in the set $S \in\{60,70, \ldots, 120\}$. Post-processing improves all average Sensitivity values by approximately $10 \%$, however, the atlases seem to slightly damage this improvement.
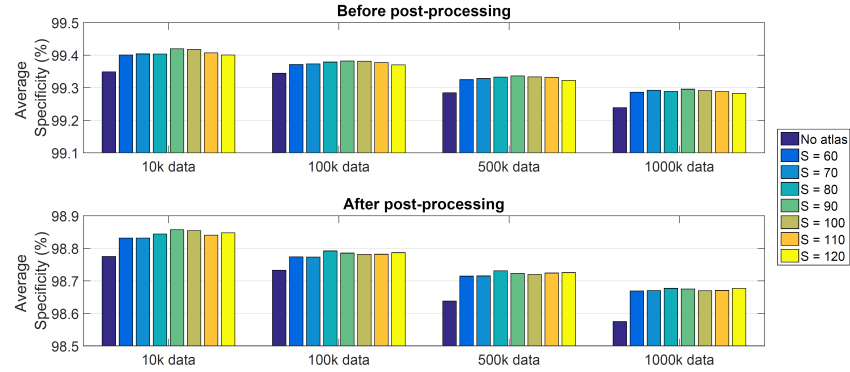

Fig. 3. Average Specificity values obtained for the 54 LG tumor volumes, before and after post-processing, without atlas and with atlas of size ranging in the set $S \in\{60,70, \ldots, 120\}$. Atlases seem to contribute to the improvement of average Specificity values by approximately $0.1 \%$.

\section{Evaluation}

Segmentation accuracy of volume $\Omega_{j}^{(2)}\left(j=1 \ldots\left|\Gamma^{(2)}\right|\right)$ is described by the number of true positive $\left(\mathrm{TP}_{j}\right)$, false positive $\left(\mathrm{FP}_{j}\right)$, true negative $\left(\mathrm{TN}_{j}\right)$ and false negative $\left(\mathrm{FN}_{j}\right)$ voxels. The most important accuracy indicators for an arbitrary test volume $\Omega_{j}^{(2)}\left(j=1 \ldots\left|\Gamma^{(2)}\right|\right)$ are:

1) Sensitivity (also referred to as true positive rate, TPR) represents the rate of identified positives among all positive voxels, and thus penalizes the occurrence of false negatives:

$$
\mathrm{TPR}_{j}=\frac{\mathrm{TP}_{j}}{\mathrm{TP}_{j}+\mathrm{FN}_{j}} .
$$

2) Specificity (also referred to as true negative rate, TNR) represents the rate of identified negatives among all negative voxels, and thus penalizes the occurrence of false positives:

$$
\mathrm{TNR}_{j}=\frac{\mathrm{TN}_{j}}{\mathrm{TN}_{j}+\mathrm{FP}_{j}}
$$

3) The Dice Score (DS) is our main accuracy indicator, which penalizes the occurrence of both false positives and false negatives:

$$
\mathrm{DS}_{j}=\frac{2 \times \mathrm{TP}_{j}}{2 \times \mathrm{TP}_{j}+\mathrm{FP}_{j}+\mathrm{FN}_{j}} .
$$

The average Dice score is the mean of the Dice scores obtained for individual volumes and is computed with the formula

$$
\overline{\mathrm{DS}}=\frac{1}{\left|\Gamma^{(2)}\right|} \sum_{j=1}^{\left|\Gamma^{(2)}\right|} \mathrm{DS}_{j},
$$

while the overall Dice score treats the test data set as a whole, and thus is defined as

$$
\widetilde{\mathrm{DS}}=\frac{2 \times \sum_{j=1}^{\left|\Gamma^{(2)}\right|} \mathrm{TP}_{j}}{\sum_{j=1}^{\left|\Gamma^{(2)}\right|}\left(2 \times \mathrm{TP}_{j}+\mathrm{FP}_{j}+\mathrm{FN}_{j}\right)} .
$$

Similarly, it is possible to extract average and overall values for sensitivity and specificity as well.

Swapping the role of the two sets $\Gamma^{(1)}$ and $\Gamma^{(2)}$ allows us to have segmentation accuracy indicators for all available MRI volumes. 


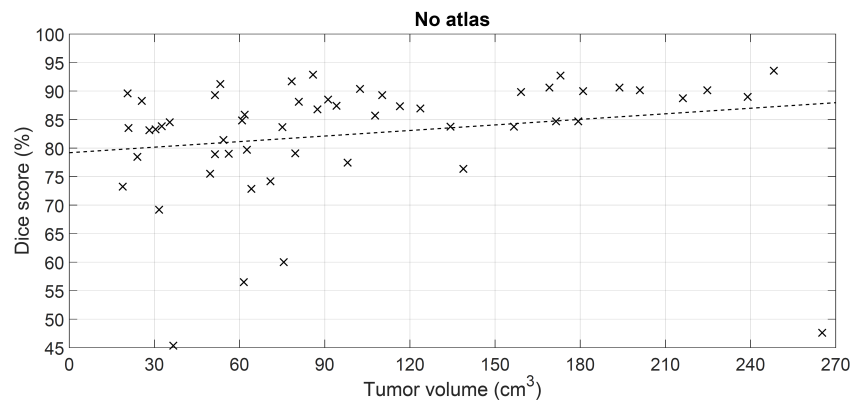

Fig. 4. Dice scores obtained for individual LG tumor volumes with no atlas. The trees of the ensemble were trained with feature vectors of $500 \mathrm{k}$ voxels.

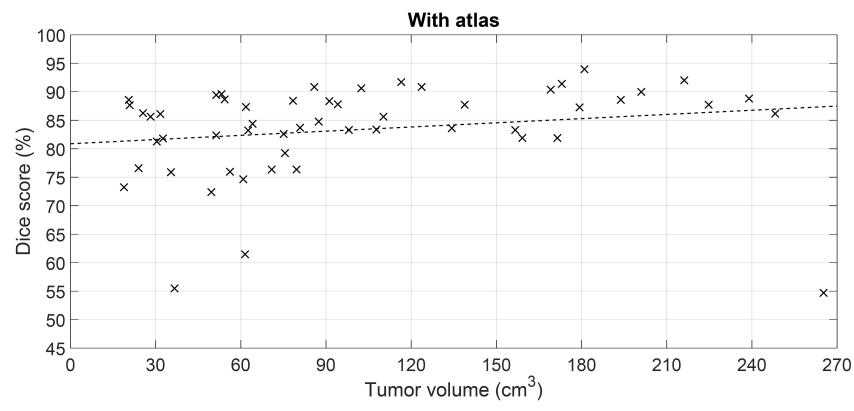

Fig. 5. Dice scores obtained for individual LG tumor volumes with the atlas of size $S=100$. The trees of the ensemble were trained with feature vectors of $500 \mathrm{k}$ voxels.

\section{RESULTS AND DISCUSSION}

The whole set of 54 LG tumor volumes was divided into two disjoint set of records, each of which played the role of train and test data set in turns. In each turn, atlases of various sizes were built using values of parameter $S$ ranging from 60 to 120 in steps of 10, while parameter $\delta$ was set to one. Ensembles were trained using feature vector sets ranging from ten thousand to one million items, out of which $93 \%$ were negatives and $7 \%$ positives. The training and testing cycle was performed for all cases and accuracy indicators recorded for each volume separately. Statistical evaluation was performed based on these recorded accuracy indicator values.

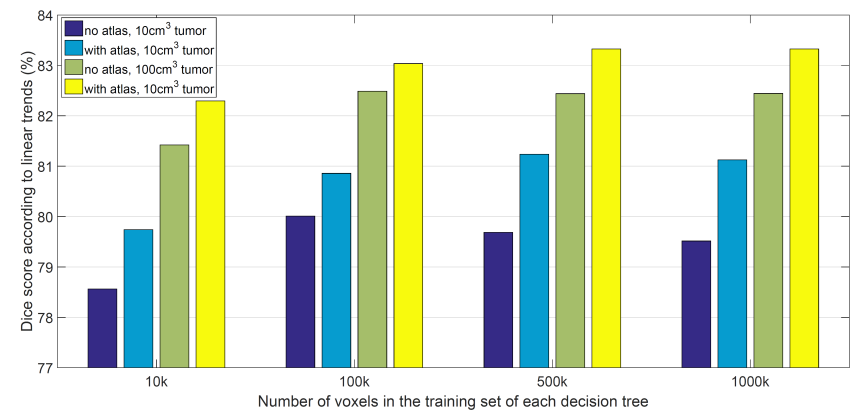

Fig. 6. Expected Dice scores for tumors of $10 \mathrm{~cm}^{3}$ and $100 \mathrm{~cm}^{3}$ computed with linear regression, at various sizes of the train data set. The atlas used here is the one with size $S=100$. The atlas improves Dice scores by $2 \%$ in case of small tumors and by $1 \%$ case of large tumors.
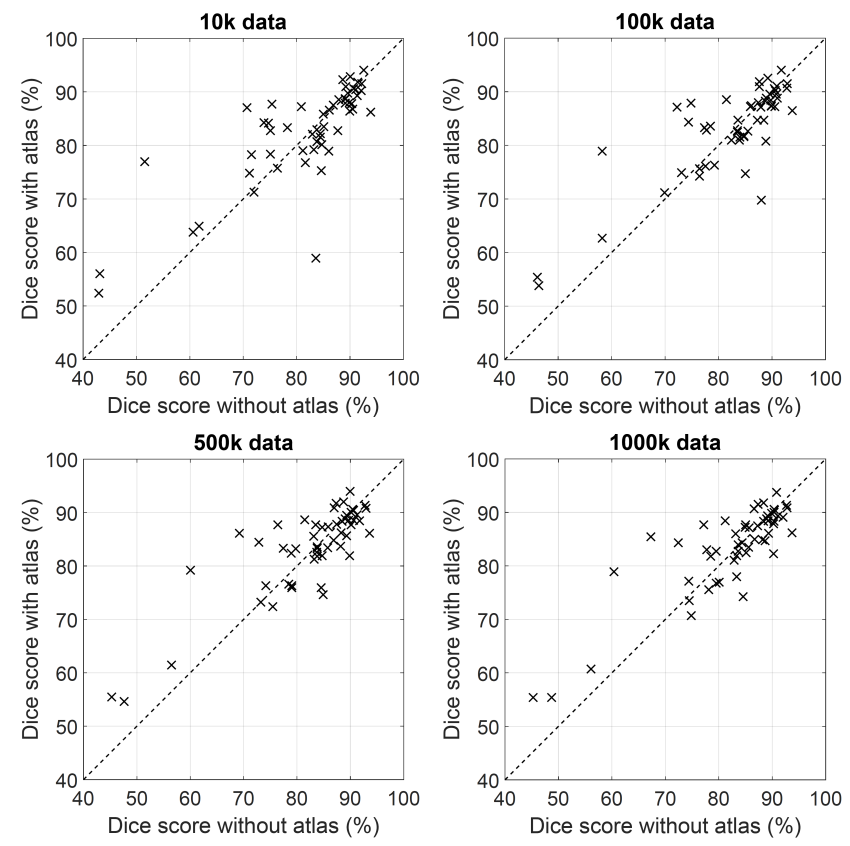

Fig. 7. Dice scores obtained for individual LG tumor volumes, values obtained with atlas $(S=100)$ plotted against values obtained without atlas. The four graphs represent the cases of ensembles whose trees were trained with $10 \mathrm{k}, 100 \mathrm{k}, 500 \mathrm{k}$, and $1000 \mathrm{k}$ feature vectors, respectively. Average Dice score is higher when the atlas is used, but individual values can be lower.

Table I reports average and overall values of the most important accuracy indicators obtained over the whole LG tumor volumes, in all the scenarios depicted in the previous paragraph. Highest indicator values are highlighted in each row of the table. Dice scores are at their highest when using the $S=100$ atlas. Sensitivity and specificity values by themselves only show that using the atlas yields the prediction of more positives, but Dice scores become definitely higher by $0.5-1.0 \%$. Figures 1,2 , and 3 exhibit the same average accuracy indicator values in graphical representation, before and after post-processing. These graphs reveal further phenomena:

1) larger train data sets provide higher DS and TPR values, and lower TNR values;

2) TPR values become higher at the ensemble output when we use the atlas, but not after post-processing, suggesting the need for an intelligent, machine learning based post-processing instead of the currently used morphological one;

3) the beneficial effect of the atlas seems to be at its highest at $90 \leq S \leq 100$.

Linear regression was employed to establish the trend of Dice scores obtained for individual LG tumor volumes, without using atlas and with using the best performing atlas. Figures 4, 5 and 6 exhibit the main findings. Figure 4 plots the obtained individual Dice scores against the true size of the tumor, and indicates the detected linear trend with the dashed line, for the no atlas case. Figure 4 plots the same thing in case of atlas usage. The difference is best visible for smaller tumors: using the atlas increases the expected Dice 
score by $1.5 \%$, it is well over $80 \%$ while it was below that when no atlas was employed. Figure 6 shows the effect of the atlas according to the linear trend for two selected sizes of the tumor, in case of various sizes of the ensemble training data set. Trends predict a $1.0-1.5 \%$ and $0.7-1.0 \%$ increase of the Dice score for a $10 \mathrm{~cm}^{3}$ and a $100 \mathrm{~cm}^{3}$-volume tumor, respectively.

Figure 7 shows the effect of the atlas upon Dice scores obtained for individual LG tumor volumes, plotting DS values achieved with atlas $(S=100)$ against $D S$ values achieved without atlas. The four graphs exhibit the cases of different train data sizes, from $10 \mathrm{k}$ to $1000 \mathrm{k}$ feature vectors for each decision tree of the ensemble. These graphs also indicate that the beneficial effect of the atlas is better visible in case of lower Dice score achieved without atlas. The use of the atlas improves the average quality of segmentation, but there are several MRI records which get higher Dice scores without atlas.

The average accuracy of the proposed atlas-based method is at the median level of deep neural network based solutions that competed at the BraTS 2017 and 2018 challenges [27]. However, our method was tested on LG volumes of the BraTS 2016 challenge only.

\section{CONCLUSIONS}

This paper introduced an ensemble learning based brain tumor segmentation algorithm enhanced by multiple atlases. An atlas was built for each feature, locally representing the average intensity of normal voxels of the train data set, together with the standard deviation of intensities. Feature values of every voxel in the train and test data set were updated using a formula that involves the extracted atlases. The updated feature values underwent the same ensemble training and testing process. Numerical evaluation revealed that the use of atlases improves the average segmentation accuracy by $1.0-1.5 \%$.

\section{REFERENCES}

[1] Y. Y. Yang, W. J. Jia and Y. N. Yang, "Multi-atlas segmentation and correction model with level set formulation for 3D brain MR images," Patt. Recogn., vol. 90, pp. 450-463, 2019.

[2] S. X. Bao, C. Bermudez, Y. K. Huo, P. Parvathaneni, W. Rodriguez, S. M. Resnick, P. F. D'Haese, M. McHugo, S. Heckers, B. M. Dawant, I. Lyu and B. A. Landman, "Registration-based image enhancement improves multi-atlas segmentation of the thalamic nuclei and hippocampal subfields," Magn. Res. Imag., vol. 59, pp. 143-152, 2019.

[3] J. Huo, J. Wu, J. W. Cao and G. H. Wang, "Supervoxel based method for multi-atlas segmentation of brain MR images," Neurolmage, vol. 175, pp. 201-214, 2018

[4] H. J. Jia, Y. Xia, Y. Song, W. D. Cai, M. Fulham and D. D. Feng, "Atlas registration and ensemble deep convolutional neural networkbased prostate segmentation using magnetic resonance imaging," Neurocomput., vol. 280, pp. 1358-1369, 2018.

[5] J. H. Zhou, Z. N. Yan, G. Lasio, J. Z. Huang, B. S. Zhang, N Sharma, K. Prado and W. D'Souza, "Automated compromised right lung segmentation method using a robust atlas-based active volume model with sparse shape composition prior in CT " Comput. Med. Imag. Graph., vol. 46, pp. 47-55, 2015.

[6] Y. M. Niu, Q. Lan and X. C. Wang, "Structured graph regularized shape prior and cross-entropy induced active contour model for myocardium segmentation in CTA images," Neurocomput., vol. 357, pp. 215-230, 2019.
[7] S. J. W. Kim, S. Seo, H. S. Kim, D. Y. Kim, K. W. Kang, J. J. Min and J. S. Lee, "Multi-atlas cardiac PET segmentation," Phys. Med., vol. 58, pp. 32-39, 2019.

[8] K. Karasawa, M. Oda, T. Kitasaka, K. Misawa, M. Fujiwara, G. W. Chu, G. Y. Zheng, D. Rueckert and K. Mori, "Multi-atlas pancreas segmentation: Atlas selection based on vessel structure," Med. Image Anal., vol. 39, pp. 18-28, 2017.

[9] O. Acosta, E. Mylona, M. Le Dain, C. Voisin, T. Lizee, B. Rigaud, C. Lafond, K. Gnep and R. De Crevoisier, "Multi-atlas-based segmentation of prostatic urethra from planning CTimaging to quantify dose distribution in prostate cancer radiotherapy," Radiotherapy and Oncology, vol. 125, pp. 492-499, 2017.

[10] H. Arabi and H. Zaidi, "Comparison of atlas-based techniques for whole-body bone segmentation," Med. Image Anal., vol. 36, pp. 98112, 2017.

[11] L. Shan, C. Zach, C. Charles and M. Niethammer, "Automatic atlasbased three-label cartilage segmentation from MR knee images," Med. Image Anal., vol. 18, pp. 1233-1246, 2014.

[12] B. Oliveira, S. Queirós, P. Morais, H. R. Torres, J. Gomes-Fonseca, J. C. Fonseca and J. Vilaça, "A novel multi-atlas strategy with dense deformation field reconstruction for abdominal and thoracic multiorgan segmentation from computed tomography," Med. Image Anal., vol. 45, pp. 108-120, 2018.

[13] D. C. T. Nguyen, S. Benameur, M. Mignotte and F. Lavoie, "Superpixel and multi-atlas based fusion entropic model for the segmentation of X-ray images," Med. Image Anal., vol. 48, pp. 58-74, 2019.

[14] M. K. Sharma, M. Jas, V. Karale, A. Sadhu and S. Mukhopadhyay, "Mammogram segmentation using multi-atlas deformable registration," Comput. Biol. Med, vol. 110, pp. 244-253, 2019.

[15] M. Cabezas, A. Oliver, X. Lladó, J. Freixenet and M. Bach Cuadra, "A review of atlas-based segmentation for magnetic resonance brain images," Comput. Meth. Prog. Bio., vol. 104, pp. e158-e177, 2011.

[16] N. Gordillo, E. Montseny and P. Sobrevilla, "State of the art survey on MRI brain tumor segmentation," Magn. Reson. Imaging, vol. 31, pp. 1426-1438, 2013.

[17] L. Sun, L. Zhang and D. Q. Zhang, "Multi-atlas based methods in brain MR image segmentation," Chin. Med. Sci. J., vol. 34, no. 2, pp. 110-119, 2019.

[18] L. Szilágyi, D. Iclănzan, Z. Kapás, Zs. Szabó, Á. Győrfi and L. Lefkovits, "Low and high grade glioma segmentation in multispectral brain MRI data", Acta Universitatis Sapientiae, Informatica, vol. 10, no. 1 , pp. $110-132,2018$

[19] Zs. Szabó, Z. Kapás, Á. Győrfi, L. Lefkovits, S. M. Szilágyi and L. Szilágyi, "Automatic segmentation of low-grade brain tumor using a random forest classifier and Gabor features", Proc. 14th International Conference on Fuzzy Systems and Knowledge Discovery, Huangshan, China, 2018, pp. 1106-1113.

[20] S. B. Akers, "Binary decision diagrams," IEEE Transactions on Computers, vol. C-27, pp. 509-516, 1978.

[21] B. H. Menze, A. Jakab, S. Bauer, J. Kalpathy-Cramer, K. Farahani, J. Kirby et al., "The multimodal brain tumor image segmentation benchmark (BRATS)," IEEE Trans. Med. Imag., vol. 34, pp. 19932024, 2015.

[22] U. Vovk, F. Pernuš and B. Likar, "A review of methods for correction of intensity inhomogeneity in MRI," IEEE Trans. Med. Imag., vol. 26, pp. 405-421, 2007.

[23] L. Szilágyi, S. M. Szilágyi, B. Benyó and Z. Benyó, "Intensity inhomogeneity compensation and segmentation of MR brain images using hybrid $c$-means clustering models", Biomed. Sign. Proc. Contr, vol. 6 , no. 1 , pp. 3-12, 2011.

[24] L. Szilágyi, S. M. Szilágyi, and B. Benyó, "Efficient inhomogeneity compensation using fuzzy $c$-means clustering models", Comput. Meth. Progr. Biomed, vol. 108, no. 1, pp. 80-89, 2012.

[25] N. J. Tustison, B. B. Avants, P. A. Cook, Y. J. Zheng, A. Egan, P. A. Yushkevich and J. C. Gee, "N4ITK: improved N3 bias correction," IEEE Trans. Med. Imag., vol. 29, no. 6, pp. 1310-1320, 2010.

[26] Á. Győrfi, Z. Karetka-Mezei, D. Iclănzan, L. Kovács and L. Szilágyi, “A study on histogram normalization for brain tumour segmentation from multispectral MR image data," Proc. Ibero-American Congress on Pattern Recognition (CIARP 2019, Havana), Lecture Notes in Computer Science, vol. 11896, pp. 375-384, 2019.

[27] S. Bakas, M. Reyes, A. Jakab, S. Bauer, M. Rempfler, A. Crimi et al., "Identifying the best machine learning algorithms for brain tumor segmentation, progression assessment, and overall survival prediction in the BRATS challenge," arXiv: 1181.02629v2, 19 Mar 2019. 